\title{
IMPRONTAS DE ESTERILLAS EN CERÁMICAS DEL BRONCE FINAL DE LA PEÑA NEGRA (CREVILLENTE, ALICANTE) (Campañas de 1983 y 1984)
}

\author{
CONCEPCIÓN PAPÍ RODES \\ Museo Arqueológico Nacional
}

\begin{abstract}
En algunos ejemplares de bases de cerámicas a mano del yacimiento del Bronce Final de La Peña Negra se observan improntas de esterillas, principalmente de estructura circular, aunque también existe algún ejemplar de tipo rectangular. El presente trabajo atiende a la descripción de este tipo de piezas y sus improntas y analiza la funcionalidad de estas esterillas, englobándolas dentro del marco de los restos de labores de cestería y esparto a lo largo de la Prehistoria más reciente.

Some fragments of pottery bases with mat's stamps from La Peña Negra (a Upper Bronze site) are presented. Generally they show round forms, but we have some examples for rectangular forms too. In this job, we attend to attribute and quality of these stamps and its functional character. We include mat's stamps in a global vision of bastketmaking during recient Prehistory.
\end{abstract}

Cuando, hace ya algunos años, entramos en contacto con los materiales del yacimiento de La Peña Negra, nos llamaron poderosamente la atención unas «huellas» que aparecían en muchos de los fragmentos de base de cerámicas a mano del horizonte del Bronce Final. En algunos casos, se apreciaban con una gran nitidez marcas de lo que parecía ser una especie de cuerdas o de trenzados más complejos, profundamente grabados sobre las superficies tiernas de las cerámicas. Las constantes tipológicas, apreciables casi a simple.vista, así como sus formas y disposición reiterativas, nos convencieron de la necesidad de someterlas a un estudio más profundo y sistemático con el fin de contestar a algunas preguntas sobre su origen y función que su observación nos planteaba, y a cuya respuesta intentaremos acercarnos a lo largo de este trabajo'.

\footnotetext{
' Queremos agradecer a Ruth Maicas su inestimable ayuda.
} 


\section{EL CONJUNTO PRE Y PROTOHISTÓRICO DE LA SIERRA DE CREVILLENTE}

La sierra de Crevillente es el marco geográfico en el que se encuadran una serie de enclaves que abarcan ya desde el Paleolítico Superior en La Ratlla del Bubo (VALVERDE y MARTI, 1984, 45), para iniciar la Edad de los Metales con el Calcolítico de Les Moreres. El Bronce Antiguo aparece atestiguado en el Pic de les Moreres, el Bronce Final en el Horizonte Peña Negra I y el orientalizante en el Horizonte Peña Negra II. Por último, el recinto fortificado de El Castellar se corresponde con un momento ibérico (GONZÁLEZ, 1983, 27 y 28).

En la campaña de 1983 se decidió retomar el sector II de La Peña Negra, sobre el que ya se había realizado sondeos en 1977 y cortes en 1979 mediante los cuales ya se constató tanto la amplitud de su secuencia estratigráfica como el importante registro de superposiciones constructivas, lo que se corroboraría no sólo en esta campaña de 1983 sino en las de los dos años siguientes (GONZÁLEZ, 1990, 15 y ss).

A las dos primeras, corresponden las cerámicas con improntas del Bronce Final, cuyo estudio ocupa estas páginas.

\section{DESCRIPCIÓN DE LOS MATERIALES}

El conjunto de los materiales cerámicos a mano con improntas tiene una serie de características comunes, en cuanto a forma y factura que ayudarán a comprender el motivo de que aparezcan como tales. Es por esta razón, que nos parece interesante incidir, al menos con un poco más de atención de lo que supone una valoración de conjunto, en la descripción de estas piezas.

*N. ${ }^{\circ}$ Inv. 6610: Fragmento de base; calidad grosera; superficie rugosa; color amarillo; pasta con núcleo; desengrasante grueso, sin decoración ${ }^{2}$. Se observa en la parte inferior una impronta de esterilla de estructura circular, cuyo punto central no es posible advertir si coincide con el de la cerámica.

${ }^{2}$ Los datos descriptivos generales sobre las cerámicas están tomados de las tramas de trabajo que realizamos durante el estudio de los materiales en el Laboratorio de Prehistoria y Arqueología de la Universidad de Alicante. Están publicadas en GONZÁLEZ, 1990, págs. 159 a 229.
*N. ${ }^{\circ}$ Inv. 6638: Fragmento de base; calidad grosera; superficie rugosa, color anaranjado-rojizo; pasta con núcleo; desengrasante grueso; sin decoración. Aparece en la parte inferior una impronta de esterilla de estructura circular, cuyo punto central coincide con el de la pieza cerámica.

*N. Inv. 6643: Fragmento de base; calidad grosera; superficie rugosa; color ocre-verdoso; pasta con núcleo; desengrasante grueso; sin decoración. Impronta de esterilla de estructura circular, cuyo punto central no podemos calcular si coincide con el de la cerámica.

*N. ${ }^{\circ}$ Inv. 6796: Fragmento de base; calidad grosera; superficie rugosa; color ocre-verdoso; pasta con núcleo; desengrasante grueso; sin decoración. Se observa en la parte inferior una impronta de esterilla de estructura circular, cuyo punto central no es posible advertir si coincide con el de la cerámica.

*N. Inv. 6821: Fragmento de base; calidad grosera; superficie rugosa; color ocre-verdoso y gris claro; pasta con núcleo; desengrasante grueso; sin decoración. Impronta de esterilla de estructura circular, cuyo punto central no es posible afirmar si coincide con el de la cerámica.

*N. ${ }^{\circ}$ Inv. 6825: Fragmento de base; calidad grosera; superficie rugosa; color ocre-verdoso; pasta con núcleo; desengrasante grueso; sin decoración. Se observa en la parte inferior una impronta de esterilla de estructura circular, cuyo punto central no nos es posible apreciar si coincide con el de la cerámica.

*N. Inv. 6860: Fragmento de base; calidad grosera; superficie rugosa; color ocre-verdoso; pasta homogénea; desengrasante grueso; sin decoración. Impronta de esterilla de estructura circular, no se puede determinar la coincidencia de su punto central con el de la cerámica.

*N. ${ }^{\circ}$ Inv. 6903: Fragmento de base; calidad grosera; superficie rugosa; color ocre-verdoso y amarillo; pasta con núcleo desengrasante grueso; sin decoración. Se observa en la parte inferior una impronta de esterilla de estructura probablemente circular, sin que podamos afirmar este punto.

*N. Inv. 6907: Fragmento de base; calidad grosera; superficie rugosa; color ocre-verdoso; pasta con núcleo; desengrasante grueso; sin decoración. Se observa en la parte inferior una impronta de esterilla de estructura circuIar, cuyo punto central coincide con el de la cerámica.

*N. Inv. 6910: Fragmento de base, calidad grosera; superficie rugosa; color ocre-verdoso; pasta con núcleo; desengrasante grueso; sin decoración. En la parte inferior tiene una impronta de esterilla de estructura circular cuyo punto central coincide perfectamente con el de la cerámica. *N. ${ }^{\circ}$ Inv. 6917: Fragmento de base; calidad grosera; superficie rugosa; color ocre-verdoso; pasta con núcleo; desengrasante grueso; sin decoración. Se observa en la parte inferior una impronta de esterilla de estructura circular, cuyo punto central coincide con el de la cerámica.

*N. ${ }^{\circ}$ Inv. 7155: Fragmento de base, calidad grosera; superficie lisa; color amarillo; pasta con núcleo; desengrasante grueso, sin decoración. Respecto a la impronta de esterilla en su parte inferior, tan sólo podemos apuntar, dado el estado de la pieza, su existencia, sin que sea posible precisar nada ni siquiera sobre su forma o estructura.

*N. ${ }^{\circ}$ Inv. 7158: Fragmento de base; calidad grosera; superficie rugosa; color ocre-verdoso; pasta con núcleo; desengrasante grueso; sin decoración. Tiene en su parte inferior una impronta de esterilla de estructura circular donde se 
aprecian dos hebras de trama. Si se realizan las mediciones pertinentes tanto de la cerámica como de la impronta, se determina con claridad la coincidencia entre el centro de ambas.

*N. ${ }^{\circ}$ Inv. 7161: Fragmento de base; calidad grosera; superficie rugosa; color ocre-verdoso; pasta con núcleo; desengrasante grueso; sin decoración. Se observa en la parte inferior una impronta de esterilla de estructura circular, cuyo punto central coincide con el de la cerámica.

*N. ${ }^{\circ}$ Inv. 7163: Fragmento de base; calidad grosera; superficie rugosa; color ocre-verdoso; pasta con núcleo; desengrasante grueso; sin decoración. En cuanto a la impronta de esterilla que tiene, sólo podemos apreciar que su estructura parece circular, si bien la impronta no se conserva en condiciones óptimas para afirmarlo categóricamente.

*N. Inv. 7164: Fragmento de base; calidad grosera; superficie rugosa; color ocre-verdoso; pasta con núcleo; desengrasante grueso; sin decoración. En este caso presenta una impronta claramente circular.

*N. ${ }^{\circ}$ Inv. 7165: Fragmento de galbo-base; calidad grosera, superficie rugosa, color ocre-verdoso; pasta con núcleo; desengrasante grueso; sin decoración. Impronta de esterilla de estructura circular.

*N. ${ }^{\circ}$ Inv. 7194: Fragmento de base; calidad grosera; superficie rugosa; color ocre-verdoso; pasta con núcleo: desengrasante grueso, sin decoración. Impronta de esterilla de estructura circular poco clara.

*N. ${ }^{\circ}$ Inv. 7206: Fragmento de base, calidad grosera, superficie rugosa; color ocre-verdoso; pasta con núcleo; desengrasante grueso; sin decoración. Impronta, mal conservada, de esterilla de estructura circular.

*N. ${ }^{\circ}$ Inv. 7217: Fragmento de base; calidad grosera; superficie rugosa; color ocre-verdoso; pasta con núcleo; desengrasante grueso; sin decoración. Impronta de esterilla de estructura circular. Al faltar la parte exterior de la base no es posible pronunciarse sobre la coincidencia de los puntos centrales.

*N. ${ }^{\circ}$ Inv. 7225: Fragmento de base; calidad grosera; superficie rugosa; color ocre-verdoso; pasta con núcleo; desengrasante grueso; sin decoración. Impronta de esterilla posiblemente circular, sin que podamos ser categóricos al respecto.

*N. ${ }^{\circ}$ Inv. 7229: Fragmento de base; calidad grosera; superficie rugosa; color ocre-verdoso; pasta con núcleo; desengrasante grueso, sin decoración. Impronta de esterilla de estructura circular. Coinciden el centro de la base y el punto de arranque de la esterilla.

*N. ${ }^{\circ}$ Inv. 7231: Fragmento de base; calidad grosera; superficie rugosa; color ocre-verdoso, pasta con núcleo desengrasante grueso; sin decoración. Impronta de esterilla de estructura circular. No es posible, dada la conservación de la cerámica apuntar nada sobre la coincidencia de los puntos centrales.

*N. ${ }^{\circ}$ Inv. 7238: Fragmento de base; calidad grosera, superficie rugosa; color ocre-verdoso; pasta homogénea; desengrasante grueso; sin decoración. Impronta de esterilla de estructura circular. No es posible establecer la coincidencia entre los centros de cerámica y esterilla.

*N. ${ }^{\circ}$ Inv. 7250: Fragmento de base; calidad grosera; superficie rugosa; color amarillo; pasta con núcleo; desengrasante grueso; sin decoración. Impronta de esterilla mal conservada y, por lo tanto, sólo podemos apuntar que de estructura circular poco clara. No es posible apuntar nada sobre la coincidencia de los puntos centrales.

*N. Inv. 7255: Fragmento de base; calidad grosera; superficie rugosa; color amarillo; pasta con núcleo; desengrasante grueso; sin decoración. Respecto a la impronta de esterilla en su parte inferior, tan sólo podemos apuntar, dado el estado de la pieza, su existencia, sin que sea posible precisar nada sobre su forma o estructura.

*N. ${ }^{\circ}$ Inv. 7256: Fragmento de base; calidad grosera; superficie rugosa; color amarillo; pasta con núcleo; desengrasante grueso; sin decoración. Impronta de esterilla de forma circular. Imposible determinar la coincidencia de puntos centrales.

*N. ${ }^{\circ}$ Inv. 7257: Fragmento de base, calidad grosera, superficie rugosa; color amarillo; pasta con núcleo; desengrasante grueso; sin decoración. Impronta de esterilla de forma circular. No es posible comprobar, por su fractura, la coincidencia de puntos centrales.

*N. ${ }^{\circ}$ Inv. 7259: Fragmento de base; calidad grosera; superficie rugosa; color amarillo; pasta con núcleo; desengrasante grueso; sin decoración. Esta es una de las mejores piezas en lo que se refiere a la impronta de esterilla que alberga: conservada con total nitidez se aprecia en ella no sólo su estructura circular, sino el nudo central del comienzo de su trabajo, que coincide perfectamente con el centro de la pieza cerámica.

*N. ${ }^{\circ}$ Inv. 7262: Fragmento de base; calidad grosera; superficie rugosa; color amarillo; pasta con núcleo; desengrasante grueso; sin decoración. Impronta muy clara de esterilla de forma circular, apreciándose total coincidencia entre su centro y el de la pieza.

*N. ${ }^{\circ}$ Inv. 7273: Fragmento de base; calidad grosera; superficie rugosa; color amarillo; pasta con varias capas; desengrasante grueso, sin decoración. Impronta de esterilla de forma circular. No se puede apreciar la coincidencia entre los centros de las piezas.

*N. ${ }^{\circ}$ Inv. 8016: Fragmento de base; calidad grosera; superficie escobillada, color amarillo; pasta con varias capas; desengrasante grueso; sin decoración. Impronta de esterilla de forma circular, en la que incluso se aprecia el nudo central del comienzo de confección. Se observa claramente la coincidencia entre los centros de las piezas

*N. ${ }^{\circ}$ Inv. 8018: Fragmento de base; calidad grosera; superficie rugosa; color ocre-verdoso; pasta con núcleo; desengrasante grueso sin decoración. La impronta de esterilla se conserva claramente observándose no sólo su estructura circular sino la coincidencia entre los puntos centrales de cerámica y esterilla.

*N. ${ }^{\circ}$ Inv. 8024: Fragmento de galbo-base; calidad grosera; superficie rugosa; color ocre-verdoso; pasta con núcleo; desengrasante grueso; sin decoración. Impronta, muy poco clara, que ni siquiera permite afirmar su forma circular.

*N. Inv. 8026: Fragmento de base; calidad grosera; superficie rugosa; color gris claro; pasta con núcleo; desengrasante grueso; sin decoración. Dado el estado de conservación de la pieza, no es posible pronunciarse sobre la coincidencia de los puntos centrales, aunque sí sobre la estructura de la esterilla, que es circular.

*N. ${ }^{\circ}$ Inv. 8027: Fragmento de base; calidad grosera; superficie rugosa; color ocre-verdoso; pasta con núcleo; desengrasante grueso; sin decoración. Dado el estado de 
conservación de la pieza, nada podemos afirmar sobre la impronta de esterilla que en ella se encuentra.

*N. Inv. 8032: Fragmento de base; calidad grosera; superficie rugosa; color ocre-verdoso; pasta con núcleo; desengrasante grueso, sin decoración. Tan sólo podemos apuntar que se aprecia una impronta de esterilla.

*N. ${ }^{\circ}$ Inv. 8033: Fragmento de base; calidad grosera; superficie rugosa; color ocre-verdoso; pasta con núcleo; desengrasante grueso; sin decoración. En contraste con las piezas antes descritas, en este caso la impronta de esterilla se conserva con una gran claridad observándose no sólo su estructura circular sino la evidente coincidencia entre los puntos centrales de cerámica y esterilla.

${ }^{*} N{ }^{\circ}$ Inv. 8035: Fragmento de base; calidad grosera; superficie rugosa; color ocre-verdoso y gris claro; pasta con núcleo; desengrasante grueso; sin decoración. Se observa impronta de esterilla de forma circular sin que sea posible añadir dato alguno sobre la coincidencia de los puntos centrales.

*N. ${ }^{\circ}$ Inv. 8039: Fragmento de base; calidad grosera; superficie rugosa; color ocre-verdoso; pasta con núcleo; desengrasante grueso; sin decoración. Impronta de tipo circular, cuyo centro coincide con el de la cerámica.

*N. ${ }^{\circ}$ Inv. 8041: Fragmento de base; calidad grosera; superficie rugosa; color gris claro; pasta homogénea; desengrasante grueso; sin decoración. Impronta de tipo circular. No es posible apreciar coincidencia de puntos centrales.

*N. ${ }^{\circ}$ Inv. 8044: Fragmento de base; calidad grosera; superficie rugosa; color ocre-verdoso; pasta con núcleo; desengrasante grueso; sin decoración. Impronta de tipo circular. No es posible añadir dato alguno sobre la coincidencia de los puntos centrales.

*N. ${ }^{\circ}$ Inv. 8271: Fragmento de base; calidad grosera; superficie rugosa; color ocre-verdoso; pasta con núcleo; desengrasante grueso; sin decoración. Nos encontramos en este caso con una estructura de esterilla diferente respecto a lo que habíamos visto hasta ahora: su estructura es rectangular. Realizada sobre una urdimbre vertical compuesta por dos pequeños juncos, la trama está formada por dos hebras retorcidas. No se aprecia en la impresión de este fragmento comienzo o remate alguno.

*N. ${ }^{\circ}$ Inv. 8316: Fragmento de base; calidad grosera; superficie rugosa; color ocre-verdoso; pasta con núcleo desengrasante grueso; sin decoración. Dado el estado de conservación del fragmento, sólo podemos apuntar la existencia de impronta de esterilla, sin entrar en más detalle.

*N. ${ }^{\circ}$ Inv. 8318: Fragmento de base; calidad grosera; superficie rugosa; color gris claro, pasta con núcleo; desengrasante grueso; sin decoración. Al igual que en la pieza 8271, estamos ante una impronta de esterilla rectangular, si bien en este caso no se aprecia con claridad la trama que parece, no obstante, muy fina. La urdimbre, en este caso, parece hecha de fibra vegetal machacada, formando un tejido que se aprecia como muy denso, donde los hilos de la urdimbre están totalmente juntos.

*N. ${ }^{\circ}$ Inv. 8424: Fragmento de base; calidad grosera; superficie rugosa; color ocre-verdoso; pasta homogénea; desengrasante grueso; sin decoración. Sólo podemos anotar que tiene una impresión de esterilla, cuya mala conservación nos impide su asignación a estructura alguna.

*N. ${ }^{\circ}$ Inv. 8453: Fragmento de base; calidad grosera; superficie rugosa; color ocre-verdoso, pasta con núcleo; desen- grasante grueso; sin decoración. Impronta de tipo circular. No es posible añadir dato alguno sobre la coincidencia de los puntos centrales.

*N. ${ }^{\circ}$ Inv. 8457: Fragmento de base; calidad grosera, superficie rugosa; color ocre-verdoso; pasta con núcleo; desengrasante grueso; sin decoración. Impronta de tipo circular. No se puede establecer la coincidencia de los puntos centrales.

*N. ${ }^{\circ}$ Inv. 8458: Fragmento de base; calidad grosera; superficie rugosa; color ocre-verdoso; pasta con varias capas; desengrasante grueso; sin decoración. Impronta de tipo circular. La fragmentación de la base no permite establecer la coincidencia o no de los puntos centrales.

*N. ${ }^{\circ}$ Inv. 8460: Fragmento de base; calidad grosera; superficie rugosa; color ocre-verdoso; pasta con varias capas; desengrasante grueso; sin decoración. Sólo podemos afirmar que tiene una impronta de esterilla, sin que podamos añadir nada más.

*N. ${ }^{\circ}$ Inv. 8461: Fragmento de base; calidad grosera; superficic rugosa; color ocre-verdoso; pasta con varias capas; desengrasante grueso; sin decoración. Impronta de esterilla de estructura circular.

*N. ${ }^{\circ}$ Inv. 8462: Fragmento de base; calidad grosera; superficie rugosa; color ocre-verdoso; pasta con núcleo desengrasante grueso; sin decoración. Tiene una impronta de esterilla, sin que podamos decir nada de su estructura, ya que está muy mal definida.

*N. ${ }^{\circ}$ Inv. 8463: Fragmento de base; calidad grosera; superficie rugosa; color ocre-verdoso, pasta con varias capas; desengrasante grueso; sin decoración. Impronta de esterilla de estructura circular.

*N. ${ }^{\circ}$ Inv. 8474: Fragmento de base; calidad grosera, superficie rugosa; color ocre-verdoso; pasta con varias capas; desengrasante grueso; sin decoración. Sólo podemos afirmar que tiene una impronta de esterilla.

*N. ${ }^{\circ}$ Inv. 8480: Fragmento de base; calidad grosera; superficie rugosa; color ocre-verdoso, pasta con núcleo desengrasante grueso; sin decoración. Tiene una impronta de esterilla, sin que podamos decir nada de su estructura.

*N. ${ }^{\circ}$ Inv. 8485: Fragmento de base; calidad grosera; superficie rugosa; color ocre-verdoso; pasta con núcleo; desengrasante grueso; sin decoración. Tiene una impronta de esterilla, pero tan mal definida que nada más podemos concluir sobre ella.

*N. ${ }^{\circ}$ Inv. 8614: Fragmento de base; calidad grosera; superficie rugosa; color ocre-verdoso; pasta con núcleo; desengrasante grueso; sin decoración. Clarísima impronta de estructura circular, cuyo punto central coincide plenamente con el de la cerámica.

*N. ${ }^{\circ}$ Inv. 8684: Fragmento de base; calidad grosera; superficie rugosa; color anaranjado-rojizo; pasta con núcleo; desengrasante grueso; sin decoración. Impronta de tipo circular. No es posible añadir dato alguno sobre la coincidencia de los puntos centrales.

*N. ${ }^{\circ}$ Inv. 8823: Fragmento de base; calidad grosera; superficie rugosa; color ocre-verdoso; pasta con núcleo; desengrasante grueso; sin decoración. Se trata de uno de los fragmentos más interesantes puesto que conserva una impresión de esterilla de estructura circular en la que se puede observar su comienzo, es decir, el nudo central del que parte toda la trama. De nuevo, como ocurre en todos los casos en que se repiten estas circunstancias, el centro de la 
esterilla coincide perfectamente con el centro de la pieza cerámica.

*N. Inv. 12037: Fragmento de base; calidad grosera, superficie rugosa; color ocre-verdoso y gris claro; pasta con varias capas; desengrasante grueso; sin decoración. Conserva una impronta de esterilla de estructura circular.

*N. Inv. 12045: Fragmento de base; calidad grosera, superficie rugosa; color ocre-verdoso; pasta con núcleo; desengrasante grueso; sin decoración. Impronta de tipo circular. No es posible añadir dato alguno sobre la coincidencia de los puntos centrales.

*N. Inv. 12914: Fragmento de base; calidad grosera; superficie rugosa; color ocre-verdoso; pasta con núcleo; desengrasante grueso, sin decoración. Tiene una impronta de esterilla, pero tan poco clara que nada más podemos concluir sobre ella.

*N. "Inv. 12915: Fragmento de base; calidad grosera; superficie rugosa; color ocre-verdoso; pasta con núcleo; desengrasante grueso; sin decoración. Tiene una impronta de esterilla, sin que podamos decir nada de su estructura.

*N. Inv. 12916: Fragmento de base; calidad grosera; superficie rugosa; color ocre-verdoso; pasta con núcleo; desengrasante grueso, sin decoración. Tiene una impronta de esterilla, sin que podamos decir nada de su estructura ya que está muy mal definida.

*N. ${ }^{\circ}$ Inv. 12917: Fragmento de base; calidad grosera; superficie rugosa; color ocre-verdoso; pasta con núcleo; desengrasante grueso; sin decoración. Impronta de tipo circular. Coinciden los puntos centrales de la esterilla y la cerámica.

\section{ANÁLISIS DE LOS TIPOS DE ESTERILLA}

Lo primero que se constata en el estudio de las improntas de esterilla, atendiendo a sus formas, tramas, y tipo de trabajo, es que los originales que dejaron su huella en las cerámicas del Bronce Final de La Peña Negra, estaban realizadas en esparto.

Este tipo de material ya había sido utilizado, desde mucho antes, para la confección no sólo de esteras, sino de toda suerte de cestos, serones, sandalias e incluso vestimentas, como veremos más adelante.

Materia prima de estos trabajos, la fibra de esparto no es sino el conjunto de filamentos que resultan de la descomposición de las hojas mediante la acción humana: de tal modo, el grosor de las fibras varía en función del desmenuzamiento previo, mientras que la longitud depende de la que tenga en origen la hoja de la planta.

Existen cuatro tipos de esparto que responden a otras tantas preparaciones de la fibra para el hilado, a grandes rasgos: dos de gran calidad, el curado o blanco y el cocido, y otros de menor valía, debido a la rapidez de su preparación, que son el oreado y el común (ALFARO, 1980).
Como ya hemos ido señando a medida que realizábamos la descripción de las piezas, es posible distinguir de forma muy clara, dos estructuras básicas de esterillas: la circular y la rectangular, quedando el grupo de improntas «probablemente» circulares como una extensión del primero, y claramente, las improntas no definidas, como grupo que nada puede aportar en cuanto a datos concretos sino acrecentar la importancia numérica de las piezas con improntas.

\section{II.1. Las esterillas circulares}

Sin duda, el modelo que se repite en mayor número de piezas es el de la esterilla circular, que aparece en más de la mitad de los ejemplares. Ha sido a través de algunas piezas donde han coincidido una serie de circunstancias favorables como la clarísima impresión, el estado de conservación, así como, incluso, la posibilidad de contemplar el nudo de arranque, lo que nos ha permitido poder identificar no sólo el tipo de trabajo realizado, sino diferentes subtipos dentro de cada una de las estructuras. De tal manera, pensamos que las esterillas circulares de La Peña Negra responden a lo que algunos autores han establecido como la «cestería cosida en espiral», donde debió ser imprescindible el uso de la aguja espartera (ALFARO, 1980, 124).

Dentro del grupo de improntas de esterillas que responden a este esquema básico, es posible establecer, incluso, distintos subtipos:

Circulares, subtipo 1 , que incluso podríamos considerar como el «standard» de las esterillas circulares, se caracteriza por una mayor separación entre sus elementos fijos (Fig. 1). Pertenecerían al mismo un total de 31 piezas ( 7259 y 12045 son las piezas donde mejor se aprecia), lo que le convierte en el subtipo más numeroso.

CirCulares, SUBTIPo 2, del cual sólo tenemos un ejemplar en la pieza 8823. Esta escasez en la representación no le resta, sin embargo, interés, ya que en ella se puede apreciar el nudo de arranque y cómo los elementos fijos que parten del mismo constan de dos hebras entrelazadas entre sí que al cabo se separan, por lo que cada elemento fijo se convierte en dos, lo que permite el aumento de tamaño de la espiral (Fig. 2).

CIRCULARES, SUBTIPO 3. Al igual que ocurre con el subtipo 2, tan sólo una pieza alberga esta clase de esteriIla circular (8614), que se caracteriza por la total proximidad de los elementos radiales (Fig. 3). No hay entre 


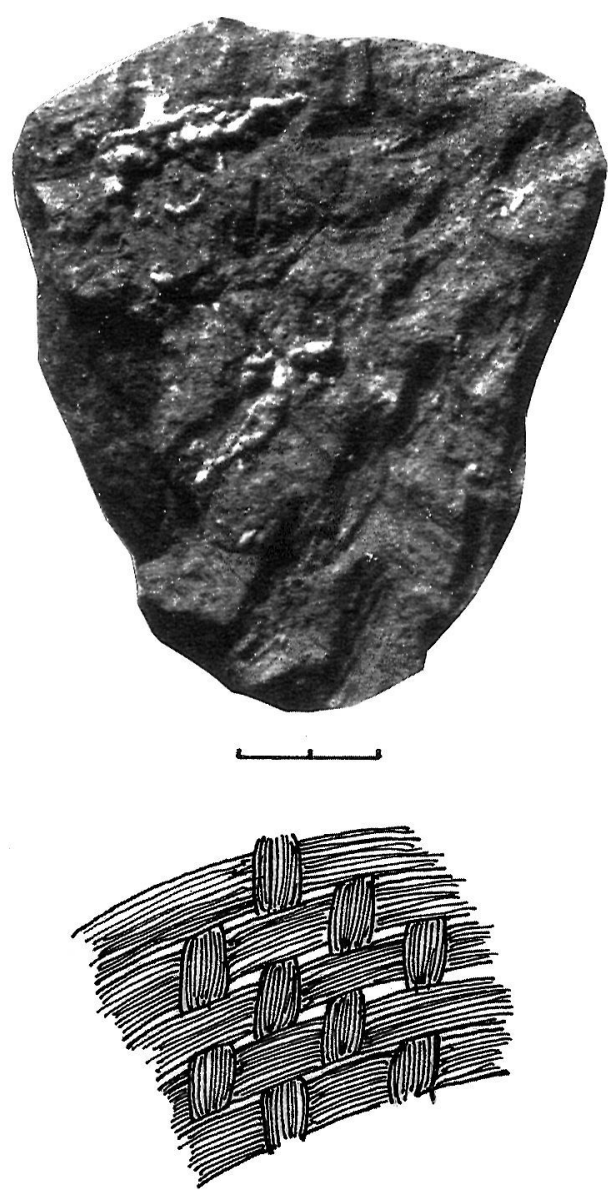

Fig.1.- Cerámica (PN. 12045) con impronta de esterilla de estructura circular, subtipo 1 , y dibujo de su entramado.

ellos, como ocurre en el subtipo 1, una separación de más o menos milímetros; aquí, los elementos radiales están «pegados» unos a otros, y su longitud en cada pasada es bastante menor que la del resto de los tipos, presentando, por lo tanto, un aspecto más «redondeado».

Circulares, subtipo 4. Los cinco ejemplares que nos han llevado a constituir este tipo presentan una característica común: el entramado se compone tanto de fibras majadas como de materia prima sin trabajar (Fig. 4). Los elementos radiales responden a las mismas características de los subtipos que hemos visto anteriormente mientras que el elemento en espiral nos ofrece un aspecto hasta ahora desconocido.

Circulares, subtipo 5. También cinco improntas componen este grupo que se caracteriza por el notable alargamiento de los elementos radiales en cada pasada (Fig. 5).
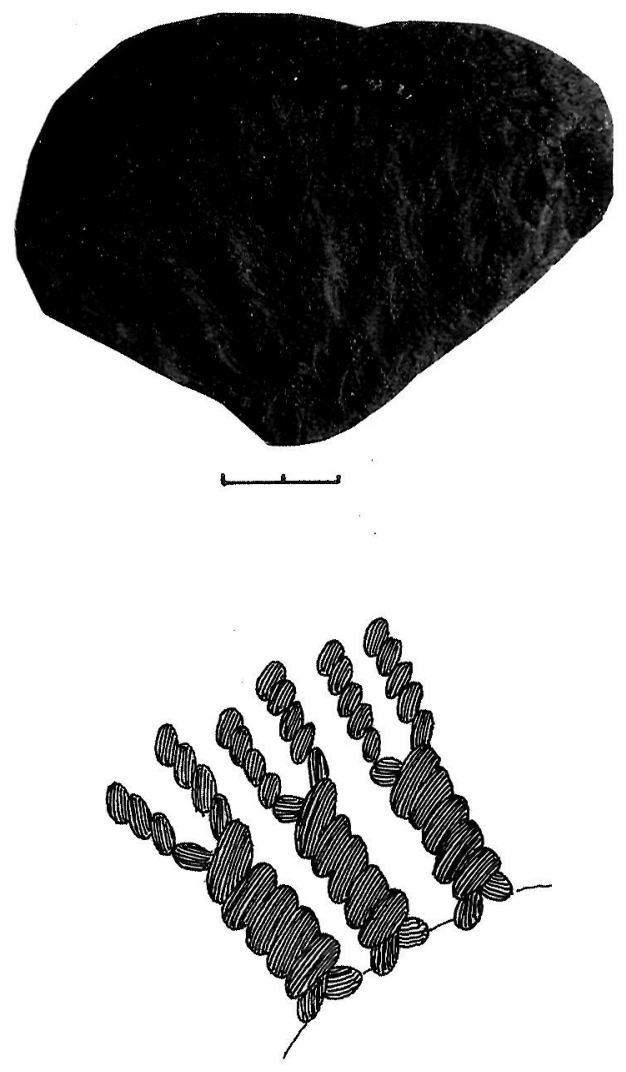

Fig.2.- Cerámica (PN. 8823) con impronta de esterilla de estructura circular, subtipo 2 , y dibujo de su entramado.

Probables improntas circulares. Como ya señalábamos en el momento de su descripción, se trata de ejemplares en los que no se puede afirmar con rotundidad que la estructura de la impronta sea circular, ya sea por lo poco nítido del grabado o por el mal estado de conservación de la pieza, aunque los rasgos que se advierten apunten, como más probable, esa posibilidad.

\section{II.2. Las esterillas rectangulares}

A pesar de contar con sólo dos fragmentos de bases que tienen una impronta de estas características, se trata de dos formas distintas de concebirla y que nos hacen, no obstante lo mínimo de la muestra, hablar de dos grupos distintos. Este tipo de entramado responde a lo que $\mathrm{C}$. Alfaro ha venido en llamar la «cestería romboidal atada» que se obtendría con unos 

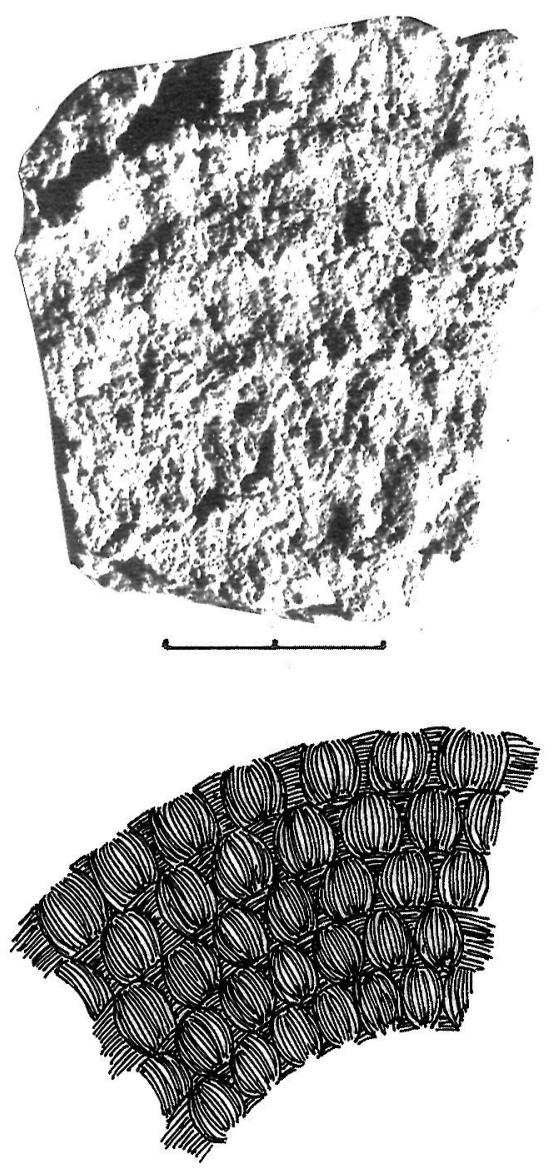

Fig.3.- Cerámica (PN. 8614) con impronta de esterilla de estructura circular, subtipo 3 , y dibujo de su entramado.

elementos fijos perpendiculares entrelazados por medio de la alternancia de dos o tres elementos móviles, que los van atando al tiempo que, al torsionarse, forman una pequeña cuerda (ALFARO, 1984, 159).

Rectangulares Subtipo 1. Aparece en la pieza 8271 , y se caracteriza porque los elementos fijos pierden su verticalidad, produciéndose un falso cruce al ser fijados por dos elementos móviles horizontales (Fig. 6). Hablamos de «falso cruce» ya que los elementos fijos unas veces se acercan y otras se mezclan uniendo y separando parte de sus fibras (ALFARO, 1984, 165)

Rectangulares, Subtipo 2. Se trata de la impronta que aparece en la base de la cerámica 8318 , donde se aprecia que el elemento fijo, de entre 1 a 1'5 cms., y por lo tanto, bastante más ancho, sirve de soporte para las fibras que forman el entramado, distribuyéndose de forma alterna en el primero y dando
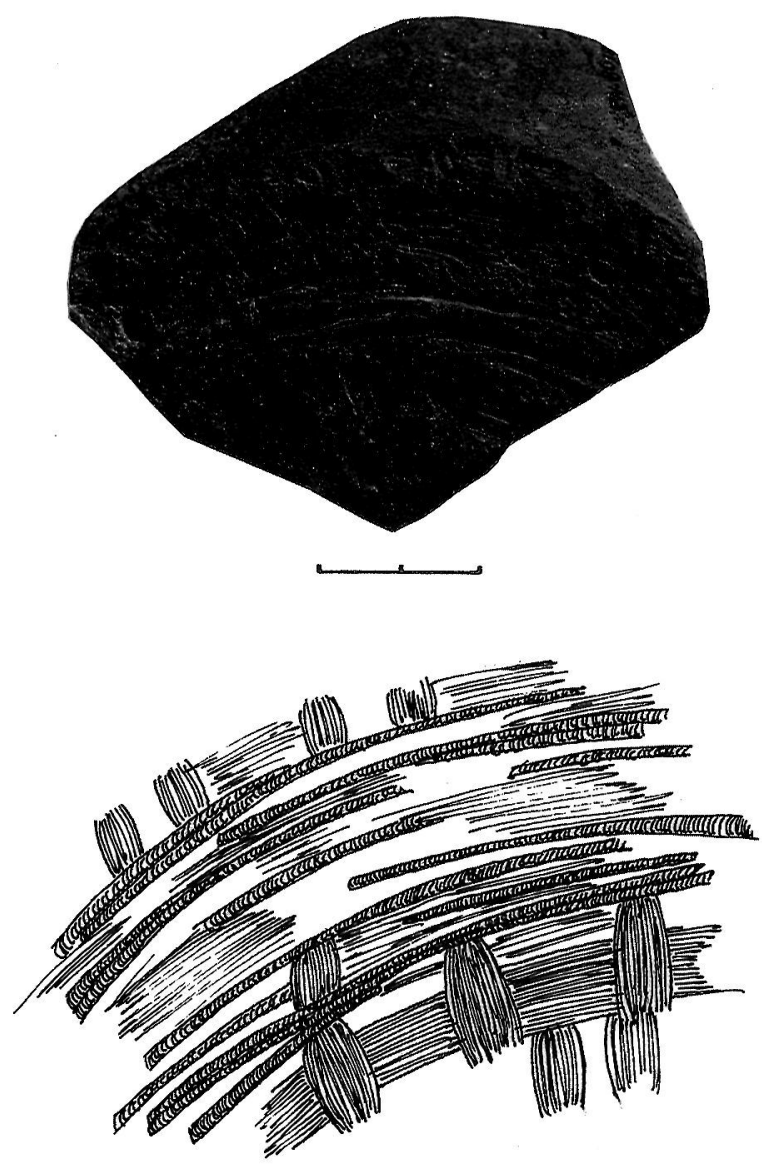

Fig.4.- Cerámica (PN. 8018) con impronta de esterilla de estructura circular, subtipo 4 , y dibujo de su entramado.

lugar, mediante una factura tensa y apretada, a una esterilla sólida y resistente (Fig. 7).

\section{II.3. Improntas indefinidas}

Existen entre las cerámicas con improntas de esterillas de La Peña Negra, catorce ejemplares de los que tan sólo se puede apuntar la presencia de unas huellas, más o menos leves, según los casos, de lo que parecer ser un entramado de esparto. El grabado poco claro y/o la mala conservación de la pieza impiden poder hacer consideración alguna más al respecto.

\section{INTERPRETACIÓN DE LAS IMPRONTAS}

Al describir las improntas de esterilla de estructura circular, hemos ido constatando el hecho de 

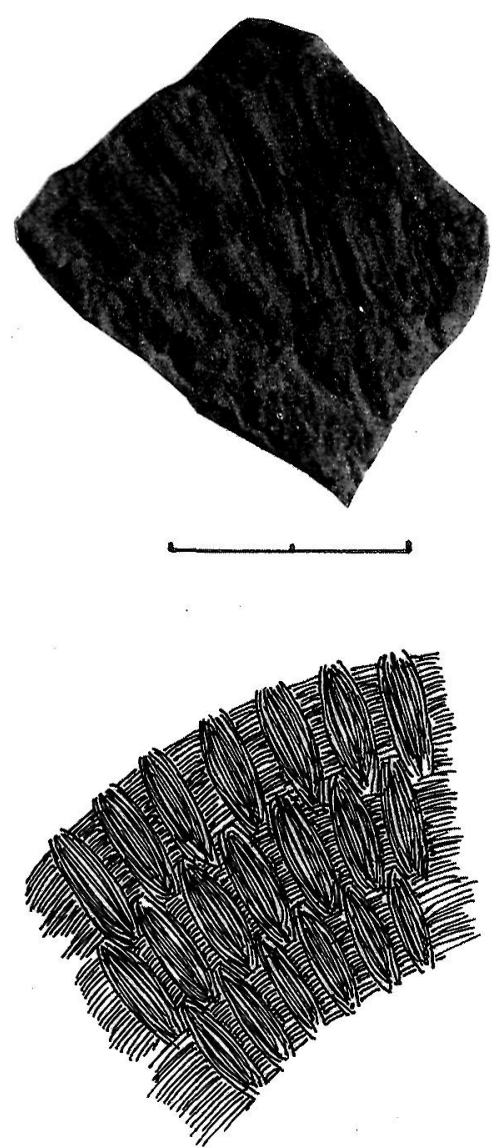

Fig. 5.- Cerámica (PN. 8457) con impronta de esterilla de estructura circular, subtipo 5, y dibujo de su entramado.

que en muchas de ellas, concretamente en dieciséis, estableciendo los cálculos pertinentes para obtener la medida de la base de la cerámica, así como observando el arco descrito por el entramado del esparto, se podía apreciar una perfecta coincidencia de los centros de ambas piezas. Este hecho, todavía era más palpable en aquellos ejemplares en los que se tenía la suerte de que la forma del fragmento de base conservado, incluyera lo que en origen sería un punto central. En todos esos casos, se ha podido constatar la presencia del nudo central o de arranque del trabajo de la esterilla.

Este dato, en modo alguno creemos que se debe al azar. Todo lo contrario, estas coincidencias patentes sólo pueden responder a una intencionalidad manifiesta.

En este momento del Bronce Final en el que el torno no ha hecho aún su aparición, sin embargo la producción de cerámicas es realmente importante. Es
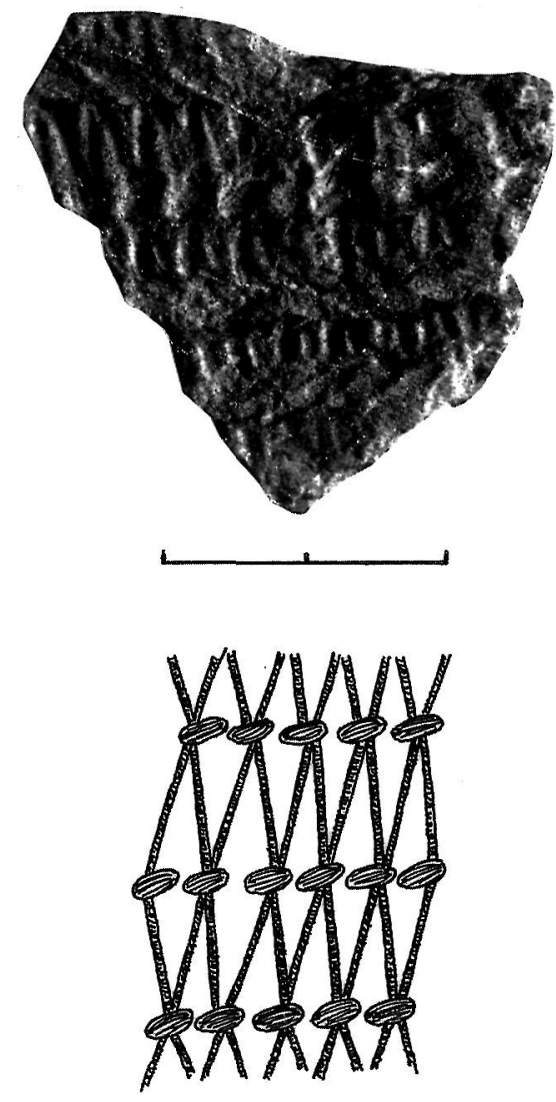

Fig. 6.- Cerámica con impronta de esterilla de estructura rectangular subtipo 1. y dibujo de su entramado.

muy posible que antes de su llegada, se utilizara una especie de «torno lento», las esterillas circulares de esparto, sobre las que se colocaría la pella de barro, obviamente en el centro, para proceder a su transformación. El peso que la pieza de cerámica está ejerciendo mientras el artesano le da forma, es lo que motiva el grabado en su base de la estera que él mismo hace girar para facilitar su trabajo.

En cuanto a las improntas rectangulares, es obvio que debían tener una finalidad distinta como podría ser la de formar parte de algún tipo de entarimado en el que, en algún momento en el que la arcilla aún estaba tierna, se depositaban las piezas cerámicas.

La presencia de improntas de esterilla en las bases de las cerámicas no es, ni mucho menos, una pauta exclusiva del Bronce Final. En el yacimiento de Campos (Almería) en la transición del Neolítico al Calcolítico se conservan piezas con clarísimas 

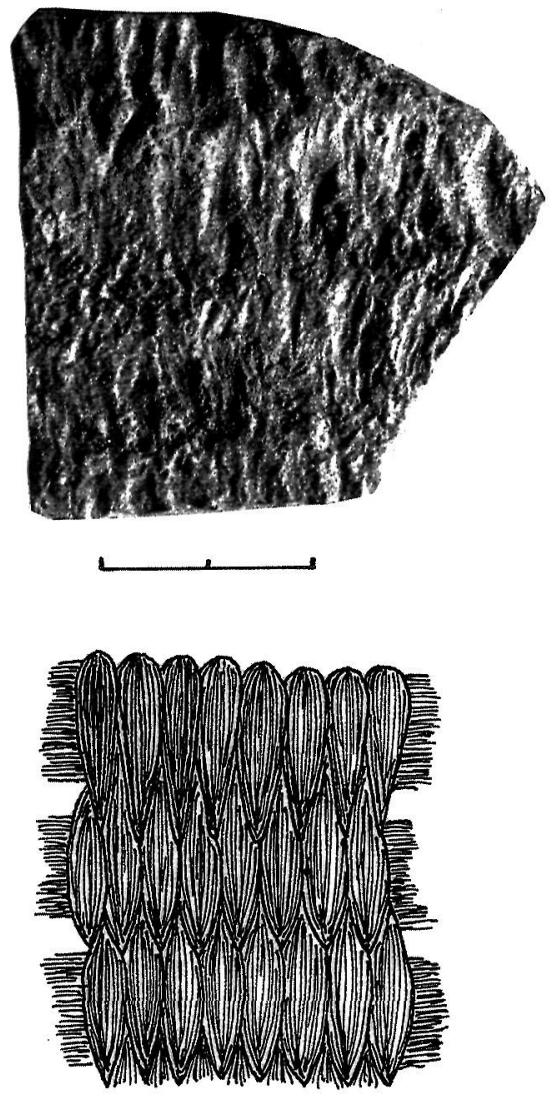

Fig.7.- Cerámica (PN. 8318) con impronta de esterilla de estructura rectangular, subtipo 2 , y dibujo de su entramado.

improntas de cestería en la base, coincidiendo, además, el punto central de ambos elementos (MARTIN y CAMALICH, 1986, 185 y ss.; CAMALICH, MARTIN y ACOSTA, 1984, 138).

No es el que proponemos para La Peña Negra, el único uso que se la ha dado a las esterillas de esparto en relación a la cerámica. En el poblado Calcolítico de El Malagón, en Granada, se han encontrado grandes fuentes de base y cocción irregular, con improntas de cestería en las bases, apuntándose entonces la posibilidad de que su gran tamaño obligara a colocar la vasija dentro de un cesto, dejándola secar en su interior antes de la cocción, de tal modo que se le quedara grabado el cesto en su zona exterior (ARRIBAS et alii, 1978, 79 y ss). Idéntica explicación se aporta para las grandes vasijas con improntas exteriores de cestería de Los Millares (ARRIBAS et alii, 1979), de Almizaraque (Almería) (DELIBES et alii, 1986, 174 y 175) y de Los Castillejos (Montefrío, Granada) (ARRIBAS y MOLINA, 1978, 90).
También han aparecido «grumos» de arcilla con improntas de estera en el yacimiento del Neolítico Antiguo conocido como Cueva 120 (Sales de Llierca, Gerona) (AGUSTI et alii, 1987,12).

Los distintos elementos de cestería realizada con esparto han sido sin duda numerosos desde momentos, al menos, neolíticos, siendo su particular necesidad medioambiental el problema de su permanencia en los yacimientos. La gran variedad de sus tipos hace que su uso no sólo esté relacionado con la elaboración de piezas cerámicas. Baste recordar el impresionante conjunto de cestería de la Cueva de los Murciélagos de Albuñol (Granada) compuesto por numerosos cestillos, cestos, esteras, cuerdas, anillas y sandalias ${ }^{3}$.

Son muchos los yacimientos desde el Neolítico hasta el Bronce en los que se han encontrado vestigios de elementos de esparto, como los de Campos (Almería), consistentes en unas cuerdas carbonizadas (SIRET, 1980, 67, lám. 10) y un cesto con semillas (MARTIN y CAMALICH, 1986, 189), mismo uso como contenedor que ya se observaba para algunos cestillos de la Cueva de los Murciélagos.

De momentos calcolíticos han aparecido en la Cueva Sagrada de Lorca (Murcia) una estera, cuerdas, e incluso rollos de esparto y esparto sin trenzar (AYALA, 1987, 9-24), más restos de cordelería en los yacimientos malagueños de la Sima de la Curra (Carratraca, Málaga) (SANCHIDRIAN, 1984-85, 240, lám. III) y en la Cueva de Nerja (JORDA et alii, 1983, 64).

En la Edad del Bronce también son frecuentes los restos de cuerdas y nudos como en Fuente Vermeja, Lugarico Viejo y El Argar (Almería), e incluso en Zapata, existe una tierra rojo-parduzca que recoge la impresión de una estera trenzada a base de coser unas tiras trenzadas con otras (SIRET, 1890, láms. 14, 16 (n. ${ }^{\circ} 67,70$ y 71), 20 (n. $\left.{ }^{\circ} 122\right)$ y 24 (n. 91 a 93$)$. También queremos recoger la impronta sobre barro de una posible estela circular procedente del poblado de

\footnotetext{
${ }^{3}$ Hemos realizado, con la colaboración de otros autores, un exhaustivo estudio de los cestillos de la Cueva de los Murciélagos, mediante reflectografía de infrarrojos, que ha proporcionado unos resultados espectaculares sobre su decoración. Este trabajo se publicará próximamente en el Homenaje que se está preparando al Dr. D. Manuel Fernández-Miranda.

${ }^{4}$ GÓNGORA, $(1868,35)$ recoge que «...dos de ellos [cestos] contenían tierra negruzca y en los restantes había mechones de cabello, valvas de moluscos y semillas de Papaver somniferum.
} 


\section{CARACTERISITICAS DEI CONUUNTO}

\begin{tabular}{|c|c|c|c|c|c|c|c|}
\hline ESTRUCTURA & $\begin{array}{l}\text { SUB- } \\
\text { TIPOS }\end{array}$ & $\begin{array}{l}\text { ESQUEMA DE } \\
\text { LABOR }\end{array}$ & \multicolumn{2}{|c|}{$\begin{array}{l}\text { CARACTERÍsTICAS } \\
\text { PRINCIPALES }\end{array}$} & $\begin{array}{l}\text { RELACIÓN DE } \\
\text { PIEZAS }\end{array}$ & $\begin{array}{l}\text { TOT. } \\
\text { PIEZ. }\end{array}$ & $\%$ \\
\hline \multirow{6}{*}{ CIRCULAR } & 1 & & অ্ & \multirow{6}{*}{ 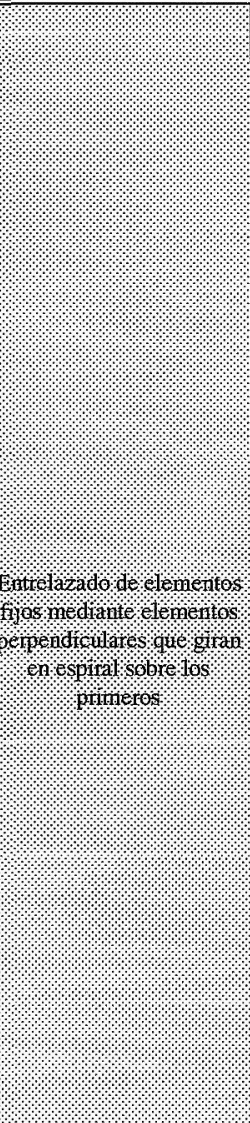 } & 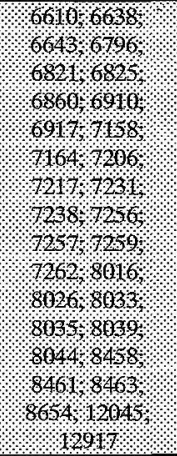 & 31 & $\sqrt{6}$ \\
\hline & 2 & & 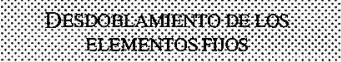 & & 188823. & $\%$ & $1 \%$ \\
\hline & 3 & & 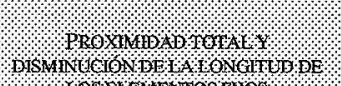 & & $861 \%$ & 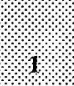 & $15 \%$ \\
\hline & 4 & & 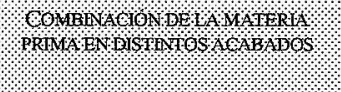 & & $6007 \%$, 10618 & 8 & \%6 \\
\hline & 5 & & 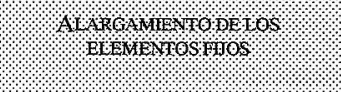 & & $801184 \%$ & 3 & 166 \\
\hline & Prob. & & 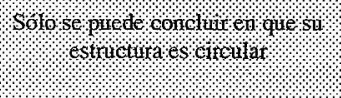 & & 600307,263 & 6 & 92 \\
\hline \multirow{2}{*}{ RECTANG. } & 1 & & 1 & \multirow{2}{*}{ 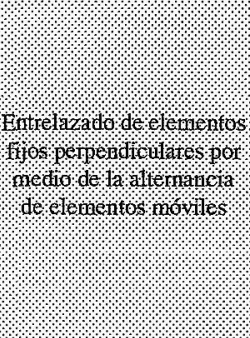 } & 827 & 1 & 15 \\
\hline & 2 & 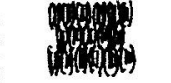 & $\sqrt[4]{1}$ y, & & 8318 & 1 & 1.1. \\
\hline INDEFINID. & & & 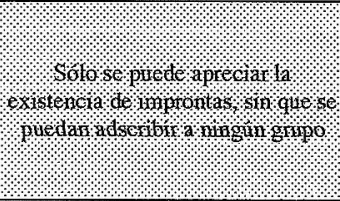 & & 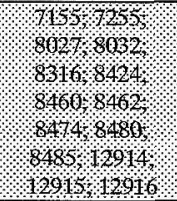 & 14 & $21 \%$ \\
\hline
\end{tabular}


Mas de Menente (Alcoy, Alicante) (PERICOT y PONSELL, 1928, lám. V, 2, citado en ALFARO, 1984, 170) y los dos pequeños fragmentos de cesta del Cabezo Redondo (Villena, Alicante) ALFARO; 1984, 170).

En cualquier caso, testimonios todos ellos de la utilización de las piezas de esparto como uno más de los elementos de la vida cotidiana, no sólo como contenedores, cuerdas o vestimentas, es decir, con una finalidad en sí mismos, sino como una herramienta de trabajo que creemos procuró una técnica «pretorno»o «torno lento» que facilitó la obtención de piezas cerámicas en el Bronce

\section{BIBLIOGRAFÍA}

AGUSTÍ, B., ALCALDE, G., BUJARCHS, F., BUXÓ, A., JUANMUNS, N., OLLER, M., ROS, T., RUEDA, J.M. y TOLEDO, A., 1987: «La Cueva 120. Un almacén del Neolítico Antiguo», Revista de Arqueología, n. ${ }^{\circ}$ 69, Madrid, págs. 7-12.

ALFARO GINER, C., 1980: «Estudio de los materiales de cestería procedentes de la cueva de Los Murciélagos (Albuñol, Granada)». Trabajos de Prehistoria, Vol. 37. Madrid, págs. 109-145.

- 1984: Tejido y cestería en la Península Ibérica. Bibliotheca Praehistorica Hispana, Vol. XXI. Madrid, 348 p.

ARRIBAS, A., y MOLINA, F., 1978: «El poblado de «Los Castillejos» en las Peñas de los Gitanos (Montefrío, Granada). Campaña de excavaciones de 1971. El corte N. 1 1 , Cuadernos de Prehistoria de la Universidad de Granada, Serie Monográfica, n. ${ }^{\circ} 3$, Granada, $280 \mathrm{p}$.

ARRIBAS, A., MOLINA, F., DE LA TORRE, F., NAJERA, T. y SAEZ, L., 1977: «El poblado eneolítico de «El Malagón de Cúllar-Baza (Granada)», Congreso Nacional de Arqueología, XIV, Vitoria, 1975, Zaragoza, 1977, págs. 319-324.

- 1978: «El poblado de la Edad del Cobre de «El Malagón» (Cúllar-Baza; Granada). Campaña de 1975», Cuadernos de Prehistoria de la Universidad de Granada, N. ${ }^{\circ} 3$, Granada, págs. 67-98.

ARRIBAS, A., MOLINA, F., DE LA TORRE, F., AGUAYO, P., y NAJERA, T., 1979. «Excavaciones en Los Millares (Santa Fe, Almería). Campañas de 1978 y 1979», Cuadernos de Prehistoria de la Universidad de Granada, N. ${ }^{\circ}$, Granada, págs. 61-96.
AYALA JUAN, M. ${ }^{a}$ M., 1987: «Enterramientos calcolíticos de la Sierra de la Tercia. Lorca. Murcia. Estudio preliminar», Anales de Prehistoria y Arqueología, 3, Secretariado de Publicaciones, Universidad de Murcia, págs. 9-24.

CAMALICH MASSIEU, M. ${ }^{a}$ D., MARTIN SOCAS, D. y ACOSTA SOSA, C., 1985: «Excavaciones en el yacimiento de Campos (Cuevas de Almanzora, Almería). Campaña de 1985», Anuario Arqueológico de Andalucía, T. II, Sevilla, págs. 134140.

DELIBES, G., FERNÁNDEZ-MIRANDA, M., FERNÁNDEZ POSSE, Ma D., y MARTÍN MORALES, C. 1986: «El poblado de Almizaraque», Actas del Coloquio «Homenaje a Luis Siret», Cuevas de Almanzora, Junio 1984, Consejería de Cultura de la Junta de Andalucía, Dirección General de Bellas Artes págs. 167-177.

GÓNGORA, M. de, 1868: Antiguedades Prehistóricas de Andalucía, Madrid, 158 p.

GONZÁLEZ PRATS, A., 1983: Estudio arqueológico del poblamiento antiguo de la Sierra de Crevillente (Alicante). Anejo I de la revista Lucentum. Universidad de Alicante, Alicante, 374 p.

-1990: Nueva luz sobre la protohistoria del Sudeste. Secretariado de Publicaciones de la Universidad de Alicante, Alicante, $357 \mathrm{p}$.

JORDÁ CERDÁ, F., JORDÁ PARDO, J.F., GONZÁLEZTABLAS, F.J., AURA TORTOSA, J.F. y SANCHIDRIÁN TORTI, J.L., 1983: «La Cueva de Nerja», Revista de Arqueología, n. ${ }^{\circ}$ 29, Madrid, págs. 57-65.

MARTÍN SOCAS, D., y CAMALICH MASSIEU, Ma D., 1986: «Las excavaciones en el poblado de Campos (Cuevas del Almanzora, Almería) y su problemática», Actas del Congreso «Homenaje a Luis Siret», Cuevas del Almanzora Junio 1984. Consejería de Cultura de la Junta de Andalucía. Dirección General de Bellas Artes, págs. 178-191.

PERICOT, L. y PONSELL, P., 1928: «El poblado de Mas de Menente (Alcoy)» Archivo de Prehistoria Levantina, 1, Valencia.

SANCHIDRIÁN TORTI, J.L., 1984-85: «Algunas bases para el estudio de los actos funerarios eneolíticos: Sima de la Curra (Carratraca, Málaga)», Zephyrus, Vol. XXXVII-XXXVIII, Salamanca, págs. 227-248.

SIRET, L. y E., 1890. Las primeras edades del metal en el Sudeste de España, Barcelona, 2 vols., 534 p.

VILLAVERDE BONILLA, V. y MARTI OLIVER, B., 1984: Les societats caçadores de la Prehistoria valenciana, Servei d'Investigació Prehistorica de la Diputació de Valencià, Valencia, $131 \mathrm{p}$. 